\title{
VALORACIÓN DE UN PROGRAMA EN SEXUALIDAD A PERSONAS CON DAÑO CEREBRAL ADQUIRIDO (DCA)
}

\author{
María Honrubia Pérez \\ Escuela de Enfermería de la Universidad de Barcelona \\ mhonrubia@ub.edu \\ Esther Sánchez Raja \\ Presidenta de la Asociación Nacional de Salud Sexual y Discapacidad (ANSSYD) \\ esanchezra@gmail.com
}

Fecha de Recepción: 1 Febrero 2018

Fecha de Admisión: 10 Abril 2018

\section{RESUMEN}

EI DCA, patología multicausal, afecta a personas jóvenes, con secuelas neurológicas que repercuten negativamente en la función sexual, en las relaciones sociales y en las de pareja de manera importante. La implantación de un programa educativo en sexualidad y la posterior valoración del mismo, cómo instrumento válido para mejorar los aspectos de la sexualidad alterados a consecuencia de la patología.

La Implantación del Programa se lleva a cabo los años 2013-16. Valoración cualitativa a través de un cuaderno de campo para describir emociones y aportaciones verbales de las personas con DCA y las profesionales. Sesión de refuerzo a los 3 meses, con la finalidad de obtener información sobre cambios en la función sexual y en las relaciones La Valoración es altamente satisfactoria de las personas con DCA y los profesionales de los centros al cuidado de estas personas, recogidos en Cuaderno de Campo.

Los programas educativos en sexualidad a personas con (DCA) son necesarios para informar y dotar de estrategias encaminadas a mantener y establecer una sexualidad saludable después del debut de la patología, por la escasez de los mismos, y la creciente demanda de información sobre la actividad sexual después de un (DCA).

Palabras claves: sexualidad; daño cerebral adquirido; programas educativos; valoración cualitativa

\section{ABSTRACT}

Evaluation of an Educational Intervention Program in Sexuality for People with Acquired Brain Injury $(\mathrm{ABI})$. 


\section{VALORACIÓN DE UN PROGRAMA EN SEXUALIDAD A PERSONAS CON DAÑO CEREBRAL ADQUIRIDO (DCA)}

The $A B I$, is a multicausal pathology that affects young people, with the presence of important neurological squeals that have a negative impact on sexual functions, social and couple relationships in an important way. The evaluation of a program on sexuality for people who have suffered an $A B I$, aims to be a valid instrument to improve the altered sexuality aspects resulted from the pathology. Implementation of the 2013-16. Subjects: 54 people, 33 men and 21 women. The qualitative evaluation is done through a field notebook, to people with $A B I$ and professionals. A session is held 3 months after implementing the program, to obtain information about changes in function and sexual relations. The evaluation is highly satisfactory for people with LCA and professionals in the institutions.

The Intervention in sexuality educational programs for people with $\mathrm{ABI}$ are considered necessary to inform and provide strategies of action aimed at maintaining and establishing a healthy sexuality after the debut of the pathology, by the shortage of the same, and the growing demand of information on sexual activity after a (DCA).

Keywords: sexuality; acquired brain injury; educational programs; qualitative assessment

\section{ANTECEDENTES}

El trabajo que se presenta, es la valoración cualitativa de un programa de intervención en sexualidad a personas adultas con Daño Cerebral Adquirido (DCA). Este programa, se considera adecuado elaborarlo e implementarlo después analizar los resultados de una investigación anterior que se llevó a cabo a nivel Nacional en España, con la colaboración de Asociaciones de Daño Cerebral Adquirido integradas en la Federación de Asociaciones de Daño Cerebral (FEDACE) y que constituyó una tesis doctoral defendida en el año 2015 en la Universidad de Barcelona. (Sánchez, E., 2015).

De las discapacidades físicas que pueden llevar asociadas alteraciones cognitivas, el Daño Cerebral Adquirido (DCA), en sus variantes: Ictus y Traumatismos Craneoencefálicos, son los que ocupan un lugar prominente tanto por el gran número de personas que lo padecen cómo por las graves secuelas que derivan de ellos. Es una patología frecuente en los países industrializados, que figura como la tercera causa de muerte en la población y la primera entre las mujeres, siendo en el mundo occidental la primera causa de incapacidad grave y crónica. Olesen, Gustavsson, Svensson, Wittchen i Jönsson, en 2012 publican un estudio sobre datos extraídos de las encuestas económicas y demográficas europeas del año 2010, en el que se refleja que 8.2 millones de personas han padecido un ictus y 3.7 millones de personas han sufrido un traumatismo craneoencefálico. Si se considera la distribución por edad y sexo, se observa que para la población europea entre 27 y 74 años, se da una incidencia del DCA de entre 318 y 372 casos por cada 100.000 varones, y entre 195 y 240 casos por cada 100.000 mujeres

En el caso de España, la Encuesta de Discapacidad, Autonomía Personal y Situaciones de Dependencia (EDAD, INE, 2008), informa de que residen 420.064 personas con (DCA), de las que el 78\% es debido a accidentes cerebro-vasculares. Los datos del Boletín del Observatorio Estatal de la Discapacidad del año 2011, confirman que el (DCA) constituye una discapacidad en aumento en nuestras sociedades y que a pesar de los avances médicos que reducen la mortalidad y el alcance de las lesiones que provocan estas patologías, el $89 \%$ de las personas con DCA necesitan de una persona de apoyo para las actividades de la vida diaria.

Estos datos hacen esencial la visibilidad social de esa patología, por la incidencia en la modificación de las conductas relacionadas con la salud, las relaciones y la sexualidad (Enguix, S., Gallardo, B., 2014).

Se define el (DCA) en el año 2008, por la National Health Injury Fundation, como una lesión traumática en el cerebro, de naturaleza no degenerativa ni congénita que puede ser debida a traumatismos como consecuencia de accidentes de tráfico, accidentes laborales, caídas lesiones o por pro- 
blemas vasculares, metabólicos, infecciones, etc..., que pueden producir una disminución 0 alteración del estado de conciencia y ocasionar un cambio a nivel cognitivo y/o físico, a la vez que una disfunción conductual y emocional (Roig Rovira, M. T., 2006). Estas deficiencias pueden presentarse de forma temporal o permanente y ser el origen de una discapacidad funcional o desajuste psicosocial con repercusiones sobre la vida de los pacientes y de sus familiares a todos los niveles. (Gifre, M., Gila, A., Pla, L., Roiga, T., Monreal-Bosch, P., 2015)

La producción científica sobre los problemas sexuales derivados de la lesión cerebral es escasa y comparativamente menor a la de los estudios realizados para proporcionar estrategias e instrumentos de rehabilitación que mejoren la Calidad de Vida de estas personas y además mucho menor que la ofertada a otras discapacidades con menor número de afectados. Este hecho hace necesario y urgente profundizar en elaborar programas de intervención en sexualidad para personas con DCA, dado el alarmante número de personas que la padecen, la severidad de sus secuelas con consecuencias en su estado físico y psicológico, y las dificultades para vivenciar plenamente su sexualidad. (Sánchez, E. 2015). Un ejemplo interesante en realización de estos programas de educación sexual para personas con DCA, es el "the sexuality education program (SEP), implementado a finales de los años 90, en todos los centros del Estado de Massachusetts (Medlar, T., 1998).

Es imprescindible, por tanto, que en los programas de rehabilitación, se integre la atención de la sexualidad. Dicha atención debe incluir, además del tratamiento específico de las disfunciones sexuales, el comportamiento sexual inadecuado, la disminución de los contactos sexuales o la desintegración de la relación y problemas sexuales, según se informa desde el Disability Services. Australia Department of Human Services, ya en el año 2008.

Por todo lo expuesto, los Programas de sexualidad deben incluir además acciones encaminadas a cambiar actitudes sociales negativas y modificar múltiples elementos contextuales que impiden la participación expedita de las personas con discapacidad en su entorno. (Castillo, 2011).

\section{OBJETIVOS}

Presentar la valoración cualitativa realizada por las profesionales de los centros en los que se realizó el programa.

Valoración cualitativa del programa por las personas con DCA.

\section{MUESTRA Y/O PARTICIPANTES}

La muestra la conforman personas con DCA de las Asociaciones: Ateneo de Castellón, Hospital Asepeyo de Sant Cugat, Asociación TRACE y Asociación ASEM de Barcelona.

Los sujetos a los que se les aplica el programa son 54 personas, de las cuales 33 son hombres y 21 son mujeres.

Los criterios de inclusión: personas con DCA, estar dado de alta desde hace más de un año, no padecer alteración cognitiva.

\section{METODOLOGÍA}

Se elabora el programa de intervención y se implanta en las asociaciones y hospital reseñados, durante los años comprendidos entre el 2013 y el 2016.

La valoración cuantitativa se realiza con posterioridad a la implantación del programa, que consta de 5 sesiones de 2 horas cada una, con un cuestionario ad hoc, tipo Lickert y preguntas abiertas. La valoración cualitativa se lleva a cabo durante las sesiones a partir de un cuaderno de campo en el que se anotan emociones, percepciones y aportaciones verbales de las personas con DCA y un cuestionario a cumplimentar por las profesionales de las asociaciones. En todos los casos son psiconeurólogas que atienden a estos pacientes. En un número total de 5 profesionales. 


\section{VALORACIÓN DE UN PROGRAMA EN SEXUALIDAD A PERSONAS CON DAÑO CEREBRAL ADQUIRIDO (DCA)}

A los 3 meses de la finalización de la intervención con el programa, se lleva a cabo una sesión de refuerzo, con valoración cualitativa con la finalidad de obtener información por parte de las personas con DCA y de las profesionales sobre posibles cambios en la función sexual, en las relaciones y en su comportamiento habitual.

\section{RESULTADOS}

Las característica sociodemográficas de las personas integrantes del Programa educativo sobre sexualidad, hacen referencia a 54 sujetos de estudio que han sufrido un DCA, desde hace más de un año y en estos momentos con alta hospitalaria, por lo que se encuentran integrados en el entorno familiar. La muestra estuvo conformada por 33 hombres (58\%9) y 21 mujeres (42\%). El rango de edad osciló entre los 29 y 60 años con un promedio de edad de 45 años. El 87\% declaran tener estudios secundarios y el 13\% universitarios. En relación a la convivencia EL $48 \%$ declaran convivir con su pareja, el $35 \%$ convive con la familia y un $17 \%$ vive de forma independiente.

Tabla 1. Frecuencia según sexo, estudios y convivencia

\begin{tabular}{|l|l|l|l|}
\hline SEXO & HOMBRES & MUJERES & TOTALES \\
\hline $\begin{array}{l}\text { NIVEL DE } \\
\text { ESTUDIOS }\end{array}$ & & & \\
\hline Básicos & & & \\
\hline Secundarios & 29 & 18 & 47 \\
\hline Universitarios & 4 & 3 & 7 \\
\hline CONVIVENCIA & & & \\
\hline Solo & 5 & 2 & 7 \\
\hline Familia & 9 & 10 & 19 \\
\hline Pareja & 17 & 9 & 26 \\
\hline
\end{tabular}

Para la obtención cualitativa de los datos, se lleva a cabo el procedimiento de utilización del cuaderno de campo, situados en el horizonte del paradigma naturalista, interpretativo o cualitativo, que utiliza los documentos elaborados durante las sesiones de intervención, como instrumento de investigación con la realización del trabajo de campo, a partir de la convicción de que "se aprenden los métodos con la práctica, de la misma forma que se aprende a nadar tirándose al agua" (Bisquerra, 2000: 261).

Desde esta perspectiva, se exponen los datos del cuaderno, donde se registran además de las actividades realizadas durante el proceso de implementación del programa de intervención, la ubicación de los centros donde se han realizado las sesiones, las sugerencias, percepciones y todo lo expresado por las personas con DCA que intervienen. Esta valoración cualitativa a partir del cuaderno de campo, importante instrumento de conocimiento del avance formativo de los grupos, además, asegura la originalidad del trabajo, permite el desarrollo del proceso poco a poco, en cada grupo y sesión, la observación de las individualidades y los grupos que transforman la información y el conocimiento en sesiones encaminadas a resolver la situación auténtica. Es importante dejar constancia además de que cuenta, el proceso de aprendizaje de la investigadora a partir de la experiencia de implantación del programa, con la consideración de ser de los pocos instrumentos que nos sirven para evidenciar, acompañar, valorar y dejar constancia progresiva de la evolución de este proceso.

La recogida de datos se lleva a cabo de forma manual con la transcripción a partir de las informaciones obtenidas en las sesiones en los discursos de los grupos, con el vaciado del cuaderno de 
campo elaborado durante la implantación del programa y sesión posterior de refuerzo. Para el análisis de datos se ha realizado un análisis de contenido temático siguiendo las etapas propuestas por Miles, M., Huberman, M., 1994 y para asegurar el rigor del estudio cualitativo se utilizaron los criterios de confiabilidad de Lincoln, Y.S., Guba, E.G., 1985.

De la información recogida durante las sesiones, emerge la valoración sobre los aspectos que se reflejan en la Tabla 2.

Tabla 2. Temas emergentes que aparecen en el cuaderno de campo de las sesiones.

\begin{tabular}{|l|l|}
\hline Sujetos que realizan el Programa & Profesionales de las Instituciones \\
\hline Interés del programa & Apoyo al Programa \\
\hline Mejora de las relaciones sexuales & Necesidad de formación en sexualidad \\
\hline Utilidad del programa & Valoración positiva \\
\hline Mejora de las relaciones & Mejora de las relaciones \\
\hline
\end{tabular}

En cuanto al Interés del Programa, queda reflejado la buena acogida por parte de los sujetos de todos los centros y la importancia de su realización.

IM005: “... nos han ido genial vuestras sesiones"

IH005: “... me ha gustado mucho, pero mucho, mucho, mucho".

IM003: "... Poder hablar de la sexualidad con personas que puedan orientarte sin vergüenza,... Me ha gustado mucho la claridad de las exposiciones",

IH004: “... el taller práctico de sensualidad"

IH009: “... El poder expresarnos y la buena información que han dado

IM006: “... La claridad cómo se habló para poder hablar públicamente comunicando tranquilidad y naturalidad para hablar del tema"

En relación a los cambios en su actividad sexual, después del programa no son muy grandes, pero si expresan modificaciones interesantes en cuanto a las relaciones sexuales

IM001: “... Me costó mucho hablar con mi esposo de sexualidad y se quedó sorprendido, le cuesta tener relaciones por si me hace daño”. "... me fui convencida de que tenía que hablar del tema y me alegré mucho de haberlo hecho".

IM002: "(una sonrisa)...Yo no lo he perdido ni lo he recuperado, yo igual o sea más, y te diría ya te digo es que en este momento es que te sientes más viva es mas es salud mental te diría sino que te sientes más viva)"

IM004: (... Hombre yo creo que si ¡Hala! ahí queda!, Ya lo he dicho y paso palabra) IH001:”... Me habéis afianzado en lo que yo pensaba antes sobre lo que eran las relaciones de pareja, pero no lo he podido practicar porque me he divorciado". IH002:"... Pero en estos meses hemos salido más juntos "... hemos ido al teatro, cine, tomar algo, a un evento con los hijos no pensar solo en mí mismo y en mi enfermedad y estar enfadado siempre".

IM005: “... no he llegado a practicar nada”,"... me visto de forma más atractiva... os oigo hablar y pienso en hacer cosas más para gustar y sentirme mejor"

En relación a la utilidad del programa hay diversas respuestas.

IH006: “... El hecho de concienciarme que aunque haya una disfunción o deficiencia la vida sexual sigue".

IH007:"... La orientación y con la profundidad que se explica el curso, la información de todo en general".

IH010.: "La muestra de los artilugios, aparatos sexuales".

IH008:"... La importancia del tocar, de comprender a la pareja como parte conjunta y no aislada". 


\section{VALORACIÓN DE UN PROGRAMA EN SEXUALIDAD A PERSONAS CON DAÑO CEREBRAL ADQUIRIDO (DCA)}

La mejora de las relaciones es evidente en el climax que se forma en el grupo, que favorece las interacciones y el unirse para realizar actividades juntos

IH24 (sonrisas) "... se tendría que haber hecho el curso antes" "... nos gustaría que durase más". "... el fin de semana iremos al Club Apolo juntos".

Un comentario muy relevante a destacar que comentan los grupos a lo largo de las sesiones, hace referencia a la falta de formación de los profesionales en materia de sexualidad y el desconocimiento de la importancia que tiene para ellos en su calidad de vida.

IH13“... tuve una depresión y cada vez me daban más medicación y mayor disfunción eréctil y esto me ocasionó mayor depresión y otra vez más medicación con más disfunción eréctil”.

En referencia a las profesionales de los Centros que intervienen en la terapia a la que se someten las personas con DCA, todas refieren que han notado cambios en cuanto a la actitud, las relaciones que se establecen en el Centro, un aumento de preguntas y cuestiones sobre sexualidad que antes no hacían y una mejor disposición hacía sus parejas, aunque las relaciones deterioradas por la patología no han mejorado después del programa. La colaboración ha sido total y han participado y asistido a las sesiones.

IP001: "... intervenir en sexualidad es factible y adecuado y debe hacerse donde los pacientes realizan las actividades diarias".

IP003: “... durante las sesiones de terapia he notado que les cuesta menos exponer dudas sobre sexualidad y hablan con más facilidad del tema"

IP002: "... hemos observado que aumentan las ganas de hacer cosas, relacionarse, salir, ir mejor vestidos y aseados".

IP004: “... durante la formación académica no se dan contenidos de sexualidad para personas con discapacidad y nos encontramos con problemas para saber cómo actuar en este ámbito".

Estos son los aspectos más relevantes a destacar del análisis del cuaderno de campo de las sesiones

\section{DISCUSIÓN}

Durante las sesiones de implantación del programa educativo, los sujetos expresaron que su actividad sexual se había alterado después del (DCA) tanto por las secuelas físicas como por las neuropsicológicas, emocionales y conductuales, tal como indican diversos estudios, (Aloni y Katz, 1999; Curcoll, M.L 2006; Roig, M.T., 2008).

Otro elemento importante que reflejan de manera recurrente en las sesiones los distintos grupos de personas que participan en el programa educativo, es el impacto negativo que tiene el DCA en la satisfacción de las relaciones de pareja. Exponen que sus parejas los consideran personas a cuidar y se convierten en cuidadores, por lo que se evidencian desajustes en las relaciones sexuales, que desembocan en la mayoría de las veces en separaciones y divorcios.

Estas mismas aportaciones están presentes en diversos estudios. (Blais, M. C. y Boisvert, J. M, 2007; Rolf, B. Gainer y Stanley Ducharme, 2010).

En el programa de intervención se pretende dar la visión de la sexualidad humana como una dimensión de la personalidad, que requiere, para poder mantener los niveles de salud sexual, un desarrollo adecuado que en la edad adulta se manifiesta en una actividad sexual frecuente, placentera y gratificante, a la vez que el establecimiento de relaciones con vinculación afectiva que implican a la vez intimidad, pasión y compromiso (Sternberg, R.J., 1988).

Una concepción amplia en el estudio de la sexualidad humana incluye tanto los aspectos cognitivos, como los afectivos, sociales y personales y transmitir actitudes positivas hacia la sexualidad y a un comportamiento sexual y afectivo "autónomo, responsable, consciente y placentero" 
(Canciano y García, 2008). Cuando esto no ocurre por causas de una patología que por sus características interfiere en el normal desarrollo de la función sexual y la intervención psicosocial, plantea una serie de problemas graves que deterioran este normal funcionamiento, pero no son tenidos en cuenta y considerados poco relevantes o relegados a un segundo plano por la importancia primera de la rehabilitación. (Castillo Cuello, 2007 y 2008).

Tal como quedó patenté en las sesiones de implantación del programa, las personas con DCA, se enfrentan cotidianamente un sinnúmero de dificultades que repercuten negativamente, sobre su sexualidad y que dificultan poder tener una expresión satisfactria de la misma. Tal cómo reflejan diversos estudios. (Chipouras et al., 1979; Master y Johnson, 1985; Kolodny,; Posse y Verdeguer, 1993; Castillo y Mena, 1995; Castillo et al., 1997; Castillo, Figueredo e Infante, 1999; Castillo Cuello, 2007).

Es muy difícil que una persona disfrute plenamente su sexualidad si no se siente, tratada como un ser sexuado, capaz de amar y ser amado, de disfrutar y hacer disfrutar con plenitud las relaciones interpersonales en general y las eróticas en particular, y sin poder, además, participar enteramente, y sin obstáculos, en el medio donde se desenvuelven. (López, F., 2006). Las personas con DCA que participaron del programa expresaban sus dificultades para poder disfrutar de su sexualidad, por haber perdido la pareja, por las secuelas fisiológicas de la enfermedad o por la disminución del deseo provocada por la misma patología.

La atención de la sexualidad, va más allá del tratamiento específico de la dificultad sexual, por lo que siempre contendrá la equiparación de oportunidades, a través de acciones sobre el ámbito social y el medio físico, (Castillo, 2011).

La mayoría de las personas de los grupos que realizan el programa, explican que no han tenido educación sexual, ni antes de la enfermedad ni después de la misma. Estas afirmaciones coinciden con un estudio de Belanger, 2009.

\section{CONCLUSIONES}

Los programas educativos en sexualidad a personas con (DCA) son necesarios para informar y dotar de estrategias encaminadas a mantener y establecer una sexualidad saludable después del debut de la patología.

Estos programas dirigidos a población adulta que ha visto modificada su función sexual y sus relaciones después del DCA, deben realizarse desde la metodología participativa, inclusiva, mediante la cual todos los miembros del grupo puedan expresarse y sentirse parte activa del mismo.

Las personas profesionales que coordinan estos grupos, han de tener una buena formación en sexualidad y conocer las características de la patología que interfieren con el normal funcionamiento de la función sexual, para poder aclarar dudas, dar informaciones veraces y responder a las demandas de las personas que integran los grupos.

La escasez de programas educativos para personas adultas que han padecido un DCA, hace necesario que se empiecen a realizar, por la gran importancia que tienen para estas personas, aspecto este verbalizado en los grupos, con la consideración de que se han de llevar a cabo cuando la situación aguda haya terminado y se encuentren de vuelta en casa, para comenzar a restablecer las pautas de comportamiento de la vida diaria.

En los grupos se verbaliza además la falta de respuesta sobre sexualidad de los profesionales especializados, aspecto este que dificulta tremendamente la normal adaptación a la pareja y a las relaciones sexuales.

Otro dato importante que surge de los grupos es la creciente demanda de información sobre la actividad sexual después de un (DCA) que expresan los pacientes y que ha de tener respuesta por 


\section{VALORACIÓN DE UN PROGRAMA EN SEXUALIDAD A PERSONAS CON DAÑO CEREBRAL ADQUIRIDO (DCA)}

parte de los profesionales que se encargan de sus cuidados y rehabilitación, para de esta manera contribuir a mejorar su calidad de vida.

\section{BIBLIOGRAFÍA}

Aloni, R., Katz, S. (1999). A review of the effect of traumatic brain injury on the human sexual response. Brain Injury, 13 (4): 269-280.

Belanger, D. (2009). Traumatic brain Injury and sexual rehabilitation. Sexologies, 18 (2): 83-85.

Bisquerra, R. (2000). Métodos de investigación educativa. Barcelona: CEAC: 261 (Blais, M. C. y Boisvert, J. M, 2007; Rolf, B. Gainer y Stanley Ducharme, 2010).

Brain Injury Association. Definición de Traumatismo Craneoencefálico. [Fecha de consulta: de abril de 2018] Disponible en: www.biausa.org

Enguix, S., Gallardo, B. (2014). El encuadre de los temas de salud: cobertura en prensa escrita del daño cerebral adquirido. Ámbitos, (Septiembre-Diciembre): [Fecha de consulta: 6 de abril de 2018] Disponible en: http://www.redalyc.org/articulo

Gifre, M., Gil, A., Pla, L., Roig, T., Monreal-Bosch, P. (2015) ¿Y después del accidente? Las necesidades psicosociales de las personas con traumatismo craneoencefálico y de sus familiares. Gac Sanit. 2015; 29(S1):60-65

Encuesta de Discapacidad, Autonomía Personal y Situaciones de Dependencia (EDAD), 2008. Madrid: INE

Medlan, T. (1998) Program Sexuality Education and Traumatic Brain Injury. Sexuality And Disability,Vol.16(1) Monografic.

Medlar, T., Medlar, J. (1990). Nursing management of sexuality issuesThe Journal of Head Trauma Rehabilitation. Vol 5 (2):46-51.

Lincoln, Y.S., Guba, E.G. (1985). Naturalistic Inquiry. Newbury Park: Sage.

López, F. (2008). Sexo y afecto en las personas con discapacidad. Madrid: Biblioteca Nueva.

Master, W.H., Johnson, V.E.(1985). Respuesta sexual Humana. Boston: Little Brown Mertens, D.M., Adams, M.J. (2004). Research and Evaluation Methods in Special Education. Thosand Oask, CA: Corwin Press

Miles, M., Huberman, M. (1994) Qualitative data analisys: An expanded sourcebook. Newbury Parck, CA: Sage.

Morse, J.M. (2003) Qualitative Health Rechearch. 13(6):833-851

Olesen, J., Gustavsson, A., Svensson, M., Wittchen, H.-U., \& Jönsson, B. (2012). The economic cost of brain disorders in Europe. European Journal of Neurology(19), 155- 162.

Sánchez, E. (2015).Salud sexual y calidad de vida en personas con Traumatismo Craneoencefálico Moderado (TCEM). Tesis Doctoral. Barcelona: Universidad de Barcelona

Sternberg, R.J. (1988). El triangulo del amor. México: Paidós 